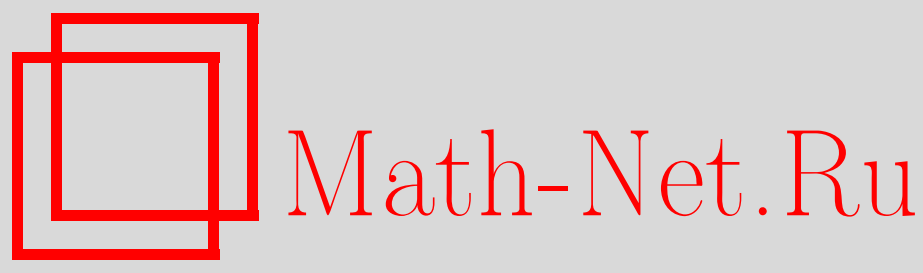

Е. В. Андронов, Влияние механизма слияния кваркглюонных струн на дальние быстротные корреляции и флуктуации, ТМФ, 2015, том 185, номер 1, 28-36

DOI: https://doi.org/10.4213/tmf8931

Использование Общероссийского математического портала Math-Net.Ru подразумевает, что вы прочитали и согласны с пользовательским соглашением http: //www . mathnet.ru/rus/agreement

Параметры загрузки:

IP: 34.239 .49 .27

26 апреля 2023 г., 13:22:26

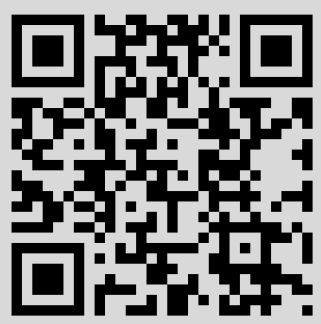




\title{
ФИЗИКА
}

Том 185, № 1

октябрь, 2015

\section{ВЛИЯНИЕ МЕХАНИЗМА СЛИЯНИЯ КВАРК-ГЛЮОННЫХ СТРУН НА ДАЛЬНИЕ БЫСТРОТНЫЕ КОРРЕЛЯЦИИ И ФЛУКТУАЦИИ}

\begin{abstract}
Множественное рождение частиц в мягких адронных взаимодействиях может быть успешно описано в рамках подхода цветовых струн, который, как полагают, со временем будет выведен из первых принципов квантовой хромодинамики. Одно из основополагающих свойств данного подхода заключается в наличии трансляционной инвариантности быстротного спектра заряженных частиц от одного источника при высоких энергиях. Эта симметрия приводит к появлению дальних быстротных корреляций через пособытийные флуктуации числа и/или типа струн. Описано поведение корреляций между множественностями $(n-n)$ и между поперечным импульсом и множественностью $\left(p_{\mathrm{t}}-n\right)$ заряженных частиц с учетом механизма формирования источников двух типов. Получено точное аналитическое выражение для коэффициента $(n-n)$-корреляции. В связи с отсутствием точного решения для коэффициента $\left(p_{\mathrm{t}}-n\right)$-корреляции предложена эффективная аппроксимация, значительно упрощающая численные вычисления. Все эти результаты проверены прямым моделированием методом Монте-Карло. Изучено влияние механизма слияния на поведение сильноинтенсивных величин, характеризующих флуктуации множественности в двух быстротных окнах.
\end{abstract}

Ключевые слова: дальние корреляции, сильноинтенсивные переменные, кварк-глюонные струны.

DOI: $10.4213 / \operatorname{tmf} 8931$

\section{1. ВВЕДЕНИЕ}

Процессы множественного рождения частиц с малыми значениями поперечного импульса $p_{\mathrm{t}}<1.5$ ГэВ/c (здесь $c$ - скорость света), лежащими в так называемой мягкой области спектра, в ядро-ядерных столкновениях не удается описывать с помощью КХД. Строгий расчет в рамках этой фундаментальной теории сильных взаимодействий возможен только для жестких партонных столкновений. Поэтому широкое распространение приобрел струнный подход [1]-[4], получивший свое

${ }^{*}$ Санкт-Петербургский государственный университет, Санкт-Петербург, Россия. E-mail: Evgeny.andronov1@gmail.com 
развитие из теории Редже-Грибова [5]-[7] и других попыток описания явлений, относящихся к непертурбативной КХД. Ключевую роль здесь играет механизм взаимодействия кварк-глюонных струн [8], [9] - протяженных по быстроте источников частиц, - который приводит к модификации распределений заряженных частиц по таким кинематическим переменным, как быстрота и поперечный импульс, а также к уменьшению средней множественности. Взаимодействие обусловлено тем, что струны имеют конечный размер в поперечной плоскости столкновения и могут перекрываться друг с другом, когда их плотность достаточно велика.

Важная особенность данного подхода заключается в том, что струна является однородным объектом по быстроте [10], т. е. имеет место трансляционная инвариантность быстротного спектра заряженных частиц от одного источника. Кроме того, части струны, разделенные в пространстве быстрот, не влияют друг на друга, т. е. рождение частиц в этих частях происходит независимо.

В связи с тем что источники дают вклад в большой диапазон быстрот, широкое развитие получил теоретический анализ дальних по быстроте корреляций [11]-[13], которые могут быть использованы для нахождения эффектов взаимодействия струн. Этот тип корреляций показывает, как связаны наблюдаемые в двух быстротных окнах, разделенных зазором, достаточным для того, чтобы пренебречь ближнекорреляционными эффектами. В качестве динамических переменных обычно выбирают экстенсивные величины (множественности заряженных частиц в быстротном окне) или интенсивные величины (среднесобытийные значения поперечного импульса заряженных частиц в быстротном окне). Корреляции между экстенсивными переменными оказываются сильно затенены флуктуациями числа кварк-глюонных струн. Корреляции интенсивных величин менее чувствительны к объемным флуктуациям, поэтому их изучение дает уникальную возможность оценки вклада коллективных эффектов.

Дополнительную информацию о взаимодействии кварк-глюонных струн может дать анализ так называемых сильноинтенсивных переменных. В работах [14], [15] показано, что для любых двух экстенсивных величин можно составить такие функции от первых двух моментов распределений этих величин, которые не зависят от среднего числа источников, а также от флуктуации их числа. Таким образом, единственным возможным источником влияния на эти переменные представляются коллективные эффекты.

В настоящей работе проведен анализ корреляций между множественностями и поперечными импульсами заряженных частиц, а также анализ сильноинтенсивной переменной, построенной из множественностей заряженных частиц.

\section{2. ОПРЕДЕЛЕНИЯ}

Величина дальних корреляций может быть охарактеризована значением коэффициентов корреляции, которые для множественностей $n_{\mathrm{B}}$ и $n_{\mathrm{F}}$ в заднем и переднем быстротных окнах, а также для среднесобытийного поперечного импульса в заднем окне $p_{\mathrm{tB}} \equiv \frac{1}{n} \sum_{i=1}^{n}\left|\mathbf{p}_{\mathrm{tB}, i}\right|$ определяются следующим образом:

$$
b_{n-n}^{\mathrm{abs}}=\frac{\left\langle n_{\mathrm{F}} n_{\mathrm{B}}\right\rangle-\left\langle n_{\mathrm{F}}\right\rangle\left\langle n_{\mathrm{B}}\right\rangle}{\left\langle n_{\mathrm{F}}^{2}\right\rangle-\left\langle n_{\mathrm{F}}\right\rangle^{2}}, \quad b_{p_{\mathrm{t}}-n}^{\mathrm{abs}}=\frac{\left\langle n_{\mathrm{F}} p_{\mathrm{tB}}\right\rangle-\left\langle n_{\mathrm{F}}\right\rangle\left\langle p_{\mathrm{tB}}\right\rangle}{\left\langle n_{\mathrm{F}}^{2}\right\rangle-\left\langle n_{\mathrm{F}}\right\rangle^{2}} .
$$

Усреднение $\langle\cdot\rangle$ в формулах (1) проводится по всем событиям. Обычно [13] коэффициенты корреляции рассматриваются в относительных переменных, т. е. после замен 
$n \rightarrow n /\langle n\rangle, p_{\mathrm{t}} \rightarrow p_{\mathrm{t}} /\left\langle p_{\mathrm{t}}\right\rangle:$

$$
b_{n-n}^{\mathrm{rel}}=\frac{\left\langle n_{\mathrm{F}}\right\rangle}{\left\langle n_{\mathrm{B}}\right\rangle} b_{n-n}^{\mathrm{abs}}, \quad b_{p_{\mathrm{t}}-n}^{\mathrm{rel}}=\frac{\left\langle n_{\mathrm{F}}\right\rangle}{\left\langle p_{\mathrm{tB}}\right\rangle} b_{p_{\mathrm{t}}-n}^{\mathrm{abs}} .
$$

Такие замены позволяют несколько ослабить зависимость корреляционных коэффициентов от ширины заднего быстротного окна.

Одна из предложенных в работах [14], [15] сильноинтенсивных переменных в случае множественностей определяется следующим образом:

$$
\Sigma_{n-n}=\frac{\left\langle n_{\mathrm{B}}\right\rangle \omega\left[n_{\mathrm{F}}\right]+\left\langle n_{\mathrm{F}}\right\rangle \omega\left[n_{\mathrm{B}}\right]-2\left(\left\langle n_{\mathrm{F}} n_{\mathrm{B}}\right\rangle-\left\langle n_{\mathrm{F}}\right\rangle\left\langle n_{\mathrm{B}}\right\rangle\right)}{\left\langle n_{\mathrm{F}}\right\rangle+\left\langle n_{\mathrm{B}}\right\rangle},
$$

где через

$$
\omega\left[n_{\mathrm{F}}\right]=\frac{\left\langle n_{\mathrm{F}}^{2}\right\rangle-\left\langle n_{\mathrm{F}}\right\rangle^{2}}{\left\langle n_{\mathrm{F}}\right\rangle}, \quad \omega\left[n_{\mathrm{B}}\right]=\frac{\left\langle n_{\mathrm{B}}^{2}\right\rangle-\left\langle n_{\mathrm{B}}\right\rangle^{2}}{\left\langle n_{\mathrm{B}}\right\rangle}
$$

обозначены приведенные дисперсии. Очевидно, что

$$
\Sigma_{n-n}=\frac{\left\langle n_{\mathrm{B}}\right\rangle \omega\left[n_{\mathrm{F}}\right]\left(1-2 b_{n-n}^{\mathrm{rel}}\right)+\left\langle n_{\mathrm{F}}\right\rangle \omega\left[n_{\mathrm{B}}\right]}{\left\langle n_{\mathrm{F}}\right\rangle+\left\langle n_{\mathrm{B}}\right\rangle} .
$$

\section{3. ФОРМАЛИЗМ}

3.1. Корреляции и флуктуации множественностей. Простейший возможный способ учета взаимодействия между источниками был предложен в модели с двумя типами источников [16], [17]. Струны первого типа образуются в результате столкновений нуклонов, струны второго типа имитируют появление новых источников в результате слияния некоторых из первых. Обозначим вероятность того, что мы имеем $N_{1}$ струн первого типа и $N_{2}$ струн второго типа, как $q\left(N_{1}, N_{2}\right)$,

$$
\sum_{N_{1}, N_{2}} q\left(N_{1}, N_{2}\right)=1
$$

Будем обозначать конфигурацию $\left\{N_{1}, N_{2}\right\}$ через $C$ и примем следующие обозначения для первых и вторых моментов этого распределения:

$$
\begin{gathered}
\sum_{C} q(C) N_{m}=\bar{N}_{m}, \quad \sum_{C} q(C) N_{m}^{2}=\overline{N_{m}^{2}}, \quad D_{N_{m}}=\overline{N_{m}^{2}}-\bar{N}_{m}^{2}, \quad m=1,2 \\
\sum_{C} q(C) N_{1} N_{2}=\overline{N_{1} N_{2}}, \quad \operatorname{cov}\left(N_{1}, N_{2}\right)=\overline{N_{1} N_{2}}-\bar{N}_{1} \cdot \bar{N}_{2} .
\end{gathered}
$$

Эти параметры характеризуют свойства конфигураций источников. В результате для множественностей имеем

$$
\begin{aligned}
& n_{\mathrm{F}}=\sum_{i=1}^{N_{1}} F_{i}^{(1)}+\sum_{j=1}^{N_{2}} F_{j}^{(2)}=n_{\mathrm{F}}^{(1)}+n_{\mathrm{F}}^{(2)}, \\
& n_{\mathrm{B}}=\sum_{i=1}^{N_{1}} B_{i}^{(1)}+\sum_{j=1}^{N_{2}} B_{j}^{(2)}=n_{\mathrm{B}}^{(1)}+n_{\mathrm{B}}^{(2)},
\end{aligned}
$$


где отдельно просуммированы вклады от каждого из источников первого и второго типов. Распределение по этим величинам тогда можно записать в следующем виде:

$$
P\left(n_{\mathrm{F}}, n_{\mathrm{B}}\right)=\sum_{C} q(C) P_{C}\left(n_{\mathrm{F}}, n_{\mathrm{B}}\right),
$$

где

$$
\begin{aligned}
P_{C}\left(n_{\mathrm{F}}, n_{\mathrm{B}}\right)= & \sum_{\left\{B_{i}^{(1)}, B_{j}^{(2)}\right\}} \delta_{n_{\mathrm{B}}, \sum_{i=1}^{N_{1}} B_{i}^{(1)}+\sum_{j=1}^{N_{2}} B_{j}^{(2)}} \sum_{\left\{F_{i}^{(1)}, F_{j}^{(2)}\right\}} \delta_{n_{\mathrm{F}}, \sum_{i=1}^{N_{1}} F_{i}^{(1)}+\sum_{j=1}^{N_{2}} F_{j}^{(2)} \times} \\
& \times \prod_{i=1}^{N_{1}} p\left(B_{i}^{(1)}, F_{i}^{(1)}\right) \prod_{j=1}^{N_{2}} p\left(B_{i}^{(2)}, F_{i}^{(2)}\right) .
\end{aligned}
$$

Здесь $p\left(B_{i}^{(1)}, F_{i}^{(1)}\right)$ и $p\left(B_{i}^{(2)}, F_{i}^{(2)}\right)$ - распределения для одного источника первого и второго типов соответственно. В случае дальних корреляций и флуктуаций предполагается, что каждая струна рождает частицы в переднее и заднее быстротные окна независимо:

$$
p\left(B_{i}^{(m)}, F_{i}^{(m)}\right)=p_{n_{\mathrm{B}}^{(m)}}\left(B_{i}^{(m)}\right) p_{n_{\mathrm{F}}^{(m)}}\left(F_{i}^{(m)}\right), \quad m=1,2,
$$

что ведет к факторизации формулы (3):

$$
P_{C}\left(n_{\mathrm{F}}, n_{\mathrm{B}}\right)=P_{C}\left(n_{\mathrm{B}}\right) P_{C}\left(n_{\mathrm{F}}\right) .
$$

Свойства одного источника определяют параметры $\bar{\mu}_{\mathrm{F}(m)}, \bar{\mu}_{\mathrm{B}}(m)-$ средние множественности в переднем и заднем окнах для частиц от одного источника первого и второго типов и соответствующие приведенные дисперсии

$$
\omega\left[\mu_{\mathrm{F}(m)}\right]=\frac{\overline{\mu_{\mathrm{F}}^{2}(m)}-\bar{\mu}_{\mathrm{F}(m)}^{2}}{\bar{\mu}_{\mathrm{F}(m)}}, \quad \omega\left[\mu_{\mathrm{B}(m)}\right]=\frac{\overline{\mu_{\mathrm{B}(m)}^{2}}-\bar{\mu}_{\mathrm{B}(m)}^{2}}{\bar{\mu}_{\mathrm{B}(m)}}, \quad m=1,2 .
$$

Рассмотрим вычисление средних значений наблюдаемых, входящих в определения коэффициента корреляции и флуктуации, на примере множественности:

$$
\left\langle n_{\mathrm{F}}\right\rangle=\sum_{C} q(C)\left\langle n_{\mathrm{F}}\right\rangle_{C}=\sum_{C} q(C)\left(N_{1} \bar{\mu}_{\mathrm{F}^{(1)}}+N_{2} \bar{\mu}_{\mathrm{F}^{(2)}}\right)=\bar{N}_{1} \bar{\mu}_{\mathrm{F}^{(1)}}+\bar{N}_{2} \bar{\mu}_{\mathrm{F}^{(2)}} .
$$

Как показано в работах [18], [19], взаимодействие цветовых полей приводит к тому, что струна нового типа, возникшая при перекрытии $\eta$ струн, обладает следующими параметрами:

$$
\bar{\mu}_{\mathrm{F}^{(2)}}=\sqrt{\eta} \bar{\mu}_{\mathrm{F}^{(1)}}, \quad \bar{\mu}_{\mathrm{B}(2)}=\sqrt{\eta} \bar{\mu}_{\mathrm{F}^{(1)}} .
$$

Используя эту связь и проводя вычисления, аналогичные (4), можно получить искомые выражения для корреляционного и флуктуационного коэффициентов

$$
\begin{aligned}
& b_{n-n}^{\mathrm{rel}}=\frac{D_{N_{1}}+\eta D_{N_{2}}+2 \sqrt{\eta} \operatorname{cov}\left(N_{1}, N_{2}\right)}{D_{N_{1}}+\eta D_{N_{2}}+2 \sqrt{\eta} \operatorname{cov}\left(N_{1}, N_{2}\right)+\left(\bar{N}_{1} \omega\left[\mu_{\mathrm{F}(1)}\right]+\sqrt{\eta} \bar{N}_{2} \omega\left[\mu_{\mathrm{F}^{(2)}}\right]\right) / \bar{\mu}_{\mathrm{F}^{(1)}}}, \\
& \Sigma_{n-n}=\omega\left[\mu_{\mathrm{F}^{(1)}}\right]+\frac{\sqrt{\eta} \bar{N}_{2}}{\bar{N}_{1}+\sqrt{\eta} \bar{N}_{2}}\left(\omega\left[\mu_{\mathrm{F}^{(2)}}\right]-\omega\left[\mu_{\mathrm{F}^{(1)}}\right]\right),
\end{aligned}
$$


записанные для краткости в случае симметричных относительно нулевой быстроты окон.

Заметим, что выражение (6) при переходе к случаю с одним типом источников совпадает с полученным ранее [20]:

$$
b_{n-n}^{\mathrm{rel}}=\frac{D_{N_{1}}}{D_{N_{1}}+\bar{N}_{1} \omega\left[\mu_{\mathrm{F}(1)}\right] / \bar{\mu}_{\mathrm{F}^{(1)}}} .
$$

Полноценный анализ поведения коэффициента корреляции (6) уже был проведен в работах [16], [17], в частности, было обнаружено немонотонное поведение данной величины в области возникновения источников второго типа.

Рассмотрим предел одного типа источников для величины (7):

$$
\Sigma_{n-n}=\omega\left[\mu_{\mathrm{F}^{(1)}}\right]
$$

Как видно, в этом пределе $\Sigma_{n-n}$ действительно проявляет свой сильноинтенсивный характер, т. е. не зависит от числа источников и его флуктуаций. Однако нормировка совпадает с требуемой в работе $[15]$ только в случае $\omega\left[\mu_{\mathrm{F}(1)}\right]=1$. В пределе, когда все первичные источники взаимодействуют, имеем

$$
\Sigma_{n-n}=\omega\left[\mu_{\mathrm{F}(2)}\right] .
$$

Выражение в правой части формулы (7) в действительности не зависит от числа источников, только если $\omega\left[\mu_{\mathrm{F}^{(2)}}\right]=\omega\left[\mu_{\mathrm{F}^{(1)}}\right]$, т. е. если дисперсия распределения по множественности при взаимодействии источников меняется пропорционально средней множественности: $D_{\mu_{\mathrm{F}(2)}}=\sqrt{\eta} D_{\mu_{\mathrm{F}(1)}}$. В этом случае две асимптотики (8) и (9) совпадают. Это условие выполняется, например, для пуассоновых источников [10], для которых $\omega\left[\mu_{\mathrm{F}^{(2)}}\right]=\omega\left[\mu_{\mathrm{F}^{(1)}}\right]=1$.

3.2. Корреляция множественности и поперечного импульса. Обозначим через $k_{i, x}^{(1)}, x=1, \ldots, B_{i}^{(1)}$, поперечные импульсы частиц, испущенных $i$-м источником первого типа в заднее быстротное окно, а через $k_{j, y}^{(2)}, y=1, \ldots, B_{j}^{(2)},-$ аналогичные импульсы частиц, испущенных $j$-м источником второго типа. Тогда плотность вероятности обнаружить событие со средним поперечным импульсом $p_{\text {tв }}$ в заднем быстротном окне при фиксированной конфигурации источников $C$ и фиксированной множественности каждого источника $\left\{B_{i}^{(1)}, B_{j}^{(2)}\right\}$ имеет вид

$$
\begin{aligned}
P_{\left\{B_{i}^{(1)}, B_{j}^{(2)}\right\}}^{C}\left(p_{\mathrm{tB}}\right)= & \int \delta\left(p_{\mathrm{tB}}-\frac{\sum_{i=1}^{N_{1}} \sum_{x=1}^{B_{i}^{(1)}} k_{i, x}^{(1)}+\sum_{j=1}^{N_{2}} \sum_{y=1}^{B_{j}^{(2)}} k_{j, y}^{(2)}}{\sum_{i=1}^{N_{1}} B_{i}^{(1)}+\sum_{j=1}^{N_{2}} B_{j}^{(2)}}\right) \times \\
& \times \prod_{i=1}^{N_{1}} \prod_{x=1}^{B_{i}^{(1)}} \rho_{1}\left(k_{i, x}^{(1)}\right) d k_{i, x}^{(1)} \prod_{j=1}^{N_{2}} \prod_{y=1}^{B_{j}^{(2)}} \rho_{2}\left(k_{j, y}^{(2)}\right) d k_{j, y}^{(2)},
\end{aligned}
$$

где

$$
\begin{gathered}
\int \rho_{1}\left(k_{i, x}^{(1)}\right) d k_{i, x}^{(1)}=\int \rho_{2}\left(k_{j, y}^{(2)}\right) d k_{j, y}^{(2)}=1 \\
\int \rho_{1}\left(k_{i, x}^{(1)}\right) k_{i, x}^{(1)} d k_{i, x}^{(1)}=\bar{k}_{1}, \quad \int \rho_{2}\left(k_{j, y}^{(2)}\right) k_{j, y}^{(2)} d k_{j, y}^{(2)}=\bar{k}_{2},
\end{gathered}
$$


что приводит к равенству

$$
\int P_{\left\{B_{i}^{(1)}, B_{j}^{(2)}\right\}}^{C}\left(p_{\mathrm{tB}}\right) d p_{\mathrm{tB}}=1 .
$$

Заметим, что для вычисления коэффициента $\left(p_{\mathrm{t}}-n\right)$-корреляции достаточно знать только первые моменты распределений по поперечному импульсу. Однако также необходимо знать совместное распределение по $p_{\text {tв }}$ и $n_{\mathrm{B}}$ :

$$
\begin{aligned}
P_{C}\left(p_{\mathrm{tB}}, n_{\mathrm{B}}\right)= & \sum_{\left\{B_{i}^{(1)}, B_{j}^{(2)}\right\}} \delta_{n_{\mathrm{B}}, \sum_{i=1}^{N_{1}} B_{i}^{(1)}+\sum_{j=1}^{N_{2}} B_{j}^{(2)}} P_{\left\{B_{i}^{(1)}, B_{j}^{(2)}\right\}}^{C}\left(p_{\mathrm{tB}}\right) \times \\
& \times \prod_{i=1}^{N_{1}} p_{n_{\mathrm{B}}^{(1)}}\left(B_{i}^{(1)}\right) \prod_{j=1}^{N_{2}} p_{n_{\mathrm{B}}^{(2)}}\left(B_{j}^{(2)}\right) .
\end{aligned}
$$

После введения обозначений

$$
\begin{aligned}
& P_{N_{1}}\left(n_{\mathrm{B}}^{(1)}\right)=\sum_{\left\{B_{i}^{(1)}\right\}} \delta_{n_{\mathrm{B}}^{(1)}, \sum_{i=1}^{N_{1}} B_{i}^{(1)}} \prod_{i=1}^{N_{1}} p_{n_{\mathrm{B}}^{(1)}}\left(B_{i}^{(1)}\right), \\
& P_{N_{2}}\left(n_{\mathrm{B}}^{(2)}\right)=\sum_{\left\{B_{j}^{(2)}\right\}} \delta_{B^{(2)}, \sum_{j=1}^{N_{2}} B_{j}^{(2)}} \prod_{j=1}^{N_{2}} p_{n_{\mathrm{B}}^{(2)}}\left(B_{j}^{(2)}\right)
\end{aligned}
$$

удается записать усреднения из выражения в числителе второй формулы в (1) в следующем виде:

$$
\begin{aligned}
\left\langle p_{\mathrm{tB}}\right\rangle= & \sum_{n_{\mathrm{B}}^{(1)}, n_{\mathrm{B}}^{(2)}} \frac{\bar{k}_{1} n_{\mathrm{B}}^{(1)}+\bar{k}_{2} n_{\mathrm{B}}^{(2)}}{n_{\mathrm{B}}^{(1)}+n_{\mathrm{B}}^{(2)}} \sum_{C} q(C) P_{N_{1}}\left(n_{\mathrm{B}}^{(1)}\right) P_{N_{2}}\left(n_{\mathrm{B}}^{(2)}\right), \\
\left\langle n_{\mathrm{F}} p_{\mathrm{tB}}\right\rangle= & \sum_{n_{\mathrm{B}}^{(1)}, n_{\mathrm{B}}^{(2)}} \frac{\bar{k}_{1} n_{\mathrm{B}}^{(1)}+\bar{k}_{2} n_{\mathrm{B}}^{(2)}}{n_{\mathrm{B}}^{(1)}+n_{\mathrm{B}}^{(2)}} \times \\
& \times \sum_{C} q(C)\left(N_{1} \bar{\mu}_{\mathrm{F}(1)}+N_{2} \bar{\mu}_{\mathrm{F}(2)}\right) P_{N_{1}}\left(n_{\mathrm{B}}^{(1)}\right) P_{N_{2}}\left(n_{\mathrm{B}}^{(2)}\right) .
\end{aligned}
$$

Вычислим теперь числитель второй формулы в (1):

$$
\begin{aligned}
& \left\langle n_{\mathrm{F}} p_{\mathrm{tB}}\right\rangle-\left\langle n_{\mathrm{F}}\right\rangle\left\langle p_{\mathrm{tB}}\right\rangle= \\
& =\sum_{n_{\mathrm{B}}^{(1)}, n_{\mathrm{B}}^{(2)}} \frac{\bar{k}_{1} n_{\mathrm{B}}^{(1)}+\bar{k}_{2} n_{\mathrm{B}}^{(2)}}{n_{\mathrm{B}}^{(1)}+n_{\mathrm{B}}^{(2)}} \sum_{C} q(C)\left(N_{1} \bar{\mu}_{\mathrm{F}^{(1)}}+N_{2} \bar{\mu}_{\mathrm{F}^{(2)}}\right) P_{N_{1}}\left(n_{\mathrm{B}}^{(1)}\right) P_{N_{2}}\left(n_{\mathrm{B}}^{(2)}\right)- \\
& \quad-\left(\sum_{n_{\mathrm{B}}^{(1)}, n_{\mathrm{B}}^{(2)}} \frac{\bar{k}_{1} n_{\mathrm{B}}^{(1)}+\bar{k}_{2} n_{\mathrm{B}}^{(2)}}{n_{\mathrm{B}}^{(1)}+n_{\mathrm{B}}^{(2)}} \sum_{C} q(C) P_{N_{1}}\left(n_{\mathrm{B}}^{(1)}\right) P_{N_{2}}\left(n_{\mathrm{B}}^{(2)}\right)\right)\left(\bar{N}_{1} \bar{\mu}_{\mathrm{F}^{(1)}}+\bar{N}_{2} \bar{\mu}_{\mathrm{F}}(2)\right) .
\end{aligned}
$$

Изменяя порядок суммирования, имеем

$$
\begin{aligned}
& \left\langle n_{\mathrm{F}} p_{\mathrm{tB}}\right\rangle-\left\langle n_{\mathrm{F}}\right\rangle\left\langle p_{\mathrm{tB}}\right\rangle=\sum_{C} q(C)\left(\left(N_{1}-\bar{N}_{1}\right) \bar{\mu}_{\mathrm{F}}(1)+\left(N_{2}-\bar{N}_{2}\right) \bar{\mu}_{\mathrm{F}(2)}\right) \times \\
& \quad \times \sum_{n_{\mathrm{B}}^{(1)}, n_{\mathrm{B}}^{(2)}} \frac{\bar{k}_{1} n_{\mathrm{B}}^{(1)}+\bar{k}_{2} n_{\mathrm{B}}^{(2)}}{n_{\mathrm{B}}^{(1)}+n_{\mathrm{B}}^{(2)}} P_{N_{1}}\left(n_{\mathrm{B}}^{(1)}\right) P_{N_{2}}\left(n_{\mathrm{B}}^{(2)}\right) .
\end{aligned}
$$

2 Теоретическая и математическая физика, т. 185, № 1, 2015 г. 
В отличие от случая корреляций множественности, данное выражение не удается привести к явной аналитической форме в связи с невозможностью усреднения дроби. Поэтому мы предлагаем рассмотреть следующее приближение, в котором знаменатель в формуле (10) заменяется на свое среднее значение по распределениям $P_{N_{1}}\left(n_{\mathrm{B}}^{(1)}\right)$ и $P_{N_{2}}\left(n_{\mathrm{B}}^{(2)}\right)$ :

$$
n_{\mathrm{B}}^{(1)}+n_{\mathrm{B}}^{(2)} \rightarrow\left\langle n_{\mathrm{B}}^{(1)}\right\rangle_{N_{1}}+\left\langle n_{\mathrm{B}}^{(2)}\right\rangle_{N_{2}} \rightarrow N_{1} \bar{\mu}_{\mathrm{F}^{(1)}}+N_{2} \bar{\mu}_{\mathrm{F}^{(2)}}
$$

Тогда формула (10) принимает вид

$$
\begin{array}{rl}
\left\langle n_{\mathrm{F}} p_{\mathrm{tB}}\right\rangle-\left\langle n_{\mathrm{F}}\right\rangle\left\langle p_{\mathrm{tB}}\right\rangle=\sum_{C} & q(C)\left(\left(N_{1}-\bar{N}_{1}\right) \bar{\mu}_{\mathrm{F}^{(1)}}+\left(N_{2}-\bar{N}_{2}\right) \bar{\mu}_{\mathrm{F}^{(2)}}\right) \times \\
\times & \frac{\bar{k}_{1} N_{1} \bar{\mu}_{\mathrm{B}^{(1)}}+\bar{k}_{2} N_{2} \bar{\mu}_{t B b}}{N_{1} \bar{\mu}_{\mathrm{B}^{(1)}}+N_{2} \bar{\mu}_{\mathrm{B}(2)}} .
\end{array}
$$

Данное приближение выглядит предпочтительно, потому что в пределе $\bar{k}_{1}=\bar{k}_{2}$ (как в модели с одним типом источников) в этом случае отсутствуют $\left(p_{\mathrm{t}}-n\right)$-корреляции, и это согласуется с общими представлениями о том, что такой тип корреляций определяется только флуктуациями типа источников.

Приближение

$$
n_{\mathrm{B}}^{(1)}+n_{\mathrm{B}}^{(2)} \rightarrow \bar{N}_{1} \bar{\mu}_{\mathrm{F}^{(1)}}+\bar{N}_{2} \bar{\mu}_{\mathrm{F}^{(2)}}
$$

выглядит менее предпочтительным, потому что в этом случае

$$
\left\langle n_{\mathrm{F}} p_{\mathrm{tB}}\right\rangle-\left\langle n_{\mathrm{F}}\right\rangle\left\langle p_{\mathrm{tB}}\right\rangle=0,
$$

т. е. теряется информация о взаимодействии источников.

Если учесть теперь связь (5) между параметрами источников двух типов и аналогичную связь $\bar{k}_{2}=\eta^{1 / 4} \bar{k}_{1}$ между средними поперечными импульсами [12], получим

$$
\left\langle n_{\mathrm{F}} p_{\mathrm{tB}}\right\rangle-\left\langle n_{\mathrm{F}}\right\rangle\left\langle p_{\mathrm{tB}}\right\rangle \approx \bar{k}_{1} \bar{\mu}_{\mathrm{F}^{(1)}}\left(\left(\bar{N}_{1}+\bar{N}_{2} \eta^{3 / 4}\right)-\left(\bar{N}_{1}+\bar{N}_{2} \eta^{1 / 2}\right)\right) \sum_{C} q(C) \frac{N_{1}+N_{2} \eta^{3 / 4}}{N_{1}+N_{2} \eta^{1 / 2}}
$$

Заметим, что оставшееся суммирование по всем возможным конфигурациям не представляет такой сложности, как генерация распада каждого источника, необходимая в (10).

Точность предлагаемого приближения была проверена с помощью прямых численных расчетов методом Монте-Карло. В работах [16], [17] был разработан монтекарловский генератор событий, соответствующий модели с двумя типами источников. Результаты его применения для вычисления коэффициента $\left(p_{\mathrm{t}}-n\right)$-корреляций в сравнении с коэффициентом, рассчитанным с приближением (11), показаны на рис. 1. На этом рисунке сплошная линия соответствует результатам генерации событий методом Монте-Карло, а точки - теоретическим результатам с приближением. По горизонтальной оси отложено среднее число $\bar{N}$ первичных источников, рассчитанное до учета взаимодействия струн.

Для оценки погрешностей вычисления весь массив генерируемых событий разделялся на 20 частей, для каждой из которых вычислялся коэффициент корреляции. Итоговое значение рассчитывалось как среднее арифметическое, а в качестве погрешности было выбрано среднеквадратичное отклонение от среднего. Как видно из рис. 1, приближение дает хорошее согласие с прямыми симуляциями методом Монте-Карло. 


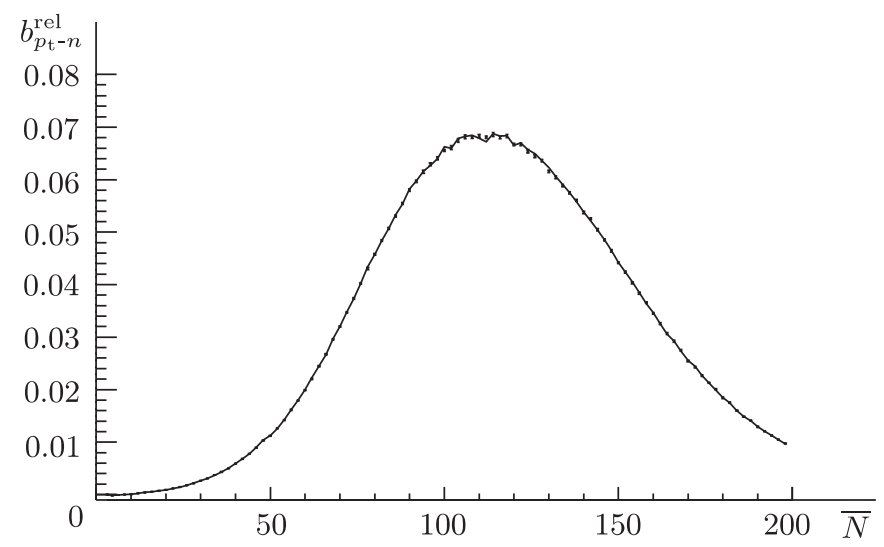

Рис. 1. Коэффициент $\left(p_{\mathrm{t}}-n\right)$-корреляций $b_{p_{\mathrm{t}}-n}^{\mathrm{rel}}$ как функция среднего числа первичных, непровзаимодействовавших источников $\bar{N}$ : результат прямых симуляций методом Монте-Карло (линия) и результат приближения (точки). Для вычисления использовались следующие параметры: $\bar{k}_{1}=0.3 \Gamma$ Э $/ c$, $\bar{\mu}_{\mathrm{F}}^{(1)}=\bar{\mu}_{\mathrm{B}(1)}=0.5, \omega\left[\mathrm{F}^{(1)}\right]=\omega\left[\mathrm{F}^{(2)}\right]=1$.

\section{4. ЗАКЛЮЧЕНИЕ}

В рамках струнного подхода проанализированы дальние корреляции и флуктуации. Получены явные аналитические выражения, когда в качестве динамических переменных выбираются множественности заряженных частиц. Переменная $\Sigma_{n-n}$ при наличии взаимодействия между кварк-глюонными струнами оказывается сильноинтенсивной только при соблюдении некоторых условий на свойства распада источников нового типа.

Для корреляций, содержащих среднесобытийный поперечный импульс, предложено использовать приближение, сравнение которого с прямыми численными расчетами методом Монте-Карло показало разумное согласие.

Благодарности. Автор благодарен В. В. Вечернину, Г. А. Феофилову и М. Газдзики за многочисленные обсуждения и замечания. Работа выполнена при поддержке Санкт-Петербургского государственного университета (грант № 11.38.197.2014).

\section{Список литературы}

[1] A. Capella, U. Sukhatme, C.-I. Tan, J. Tran Thanh Van, Phys. Lett. B, 81:1 (1979), 68-74.

[2] A. Capella, U. Sukhatme, C.-I. Tan, J. Tran Thanh Van, Phys. Rep., 236:4-5 (1994), $225-329$.

[3] A. B. Kaidalov, Phys. Lett. B, 116:6 (1982), 459-463.

[4] A. B. Kaidalov, K. A. Ter-Martirosyan, Phys. Lett. B, 117:3-4 (1982), 247-251.

[5] В. Н. Грибов, ЖЭТФ, 53:2 (1968), 654-672.

[6] K. Werner, Phys. Rep., 232:2-5 (1993), 87-299.

[7] K. Werner, T. Hirano, Iu. Karpenko, T. Pierog, S. Porteboeuf, M. Bleicher, S. Haussler, Nucl. Phys. B: Proc. Suppl., 196 (2009), 36-43.

[8] M. A. Braun, C. Pajares, Phys. Lett. B, 287:1-3 (1992), 154-158.

[9] M. A. Braun, C. Pajares, Nucl. Phys. B, 390:2 (1993), 542-558. 
[10] M. A. Braun, C. Pajares, V. V. Vechernin, Phys. Lett. B, 493:1-2 (2000), 54-64.

[11] N. S. Amelin, N. Armesto, M. A. Braun, E. G. Ferreiro, C. Pajares, Phys. Rev. Lett., 73:21 (1994), 2813-2816.

[12] M. A. Braun, R. S. Kolevatov, C. Pajares, V. V. Vechernin, Eur. Phys. J. C, 32:4 (2004), $535-546$.

[13] В. В. Вечернин, Р. С. Колеватов, ЯФ, 70:10 (2007), 1846-1857.

[14] M. I. Gorenstein, M. Gazdzicki, Phys. Rev. C, 84:1 (2011), 014904, 5 pp., arXiv: 1101.4865.

[15] M. Gazdzicki, M. I. Gorenstein, M. Mackowiak-Pawlowska, Phys. Rev. C, 88:2 (2013), 024907, 9 pp.

[16] E. Andronov, V. Vechernin, PoS(QFTHEP 2013), 2014, 054, 7 pp.

[17] E. Andronov, V. Vechernin, PoS(Baldin ISHEPP XXII), 2015, 068, 14 pp.

[18] T. S. Biro, H. B. Nielsen, J. Knoll, Nucl. Phys. B, 245 (1984), 449-468.

[19] A. Bialas, W. Czyz, Nucl. Phys. B, 267:1 (1986), 242-252.

[20] V. V. Vechernin, Long-Range rapidity correlations in the model with independent emitters, arXiv: 1012.0214. 